\title{
0173 INJURIES ASSOCIATED WITH MOTION-SENSING ELECTRONIC GAME CONSOLES: DATA FROM 2007 TO 2008
}

C Jones, ${ }^{*}$ B Hammig, J Henry Correspondence: Department of Health, University of Arkansas, 308 HPER Building Fayetteville, AR 72701, USA

10.1136/ip.2010.029215.173

Computer games and software represent a 10 billion dollar market annually. Approximately $65 \%$ of Americans have engaged in gaming systems in 2008. With use comes the issue of misuse or abuse of the systems that could lead to injury. As the game systems become more popular and extend the features to include more physical movement, will there be a rise in injuries and morbidity? One popular form of game system uses motion-sensing technology to mimic physical movement. The purpose of this study is to review recent statistics on injuries related to electronic gaming systems and highlight opportunities for health educators to promote safe use of the systems. Methods included obtaining data from the National Electronic Injury Surveillance System (NEISS) for product code 0557 for the year 2008. Results indicate that injuries from electronic gaming systems increased by almost $214 \%$ from 2007 to 2008 going from 21 to 45 injuries. The mean age for injury was 15 and an equal risk for gender. Most injuries involved the Head/ Neck region, Hand/Finger or the Arm. The major cause of the injury was due to overuse of the game system or being hit by the game system remote. Opportunities for education involve information on the risk for injury from extended use as well as establishing a play area that provides plenty of room to move. Continued surveillance is recommended to determine the trend for injury as the game systems become more popular. 